\title{
THE OUTBURSTS AND ORBIT OF THE ACCRETING PULSAR GS 1843-02 = 2S 1845-024
}

\author{
Mark H. Finger, ${ }^{1,2}$ Lars Bildsten, ${ }^{3}$ Deepto Chakrabarty, ${ }^{4}$ Thomas A. Prince, ${ }^{5}$ D. Matthew Scott, ${ }^{1,2}$ \\ Colleen A. Wilson, ${ }^{1}$ Robert B. Wilson, ${ }^{1}$ AND S. NAN Zhang ${ }^{1,6}$ \\ Received 1998 September 28; accepted 1999 December 29
}

\begin{abstract}
We present observations of a series of 10 outbursts of pulsed hard X-ray flux from the transient 10.6 $\mathrm{mHz}$ accreting pulsar GS 1843-02, using the Burst and Transient Source Experiment on the Compton Gamma Ray Observatory. These outbursts occurred regularly every 242 days, coincident with the ephemeris of the periodic transient GRO J1849-03, which has recently been identified with the $S A S 3$ source 2S 1845-024. Our pulsed detection provides the first clear identification of GS $1843-02$ with $2 \mathrm{~S}$ 1845-024. We present a pulse timing analysis that shows that the 2S 1845-024 outbursts occur near the periastron passage of the neutron star's highly eccentric $(e=0.88 \pm 0.01) 242.18 \pm 0.01$ day period binary orbit about a high-mass $\left(M_{c}>7 M_{\odot}\right)$ companion. The orbit and transient outburst pattern strongly suggest that the pulsar is in a binary system with a Be star. Our observations show a long-term spin-up trend, with most of the spin-up occurring during the outbursts. From the measured spin-up rates and inferred luminosities we conclude that an accretion disk is present during the outbursts.

Subject headings: accretion, accretion disks — binaries: general — pulsars: individual (GS 1843-02) pulsars: individual (2S 1845-024) — stars: emission-line, Be — stars: neutron
\end{abstract}

\section{INTRODUCTION}

The pulsar GS 1843-02 was discovered with Ginga in 1988 (Makino 1988) during a galactic plane scan conducted as part of a search for transient pulsars (Koyama et al. 1990a). These observations found six new transient X-ray sources near galactic longitude $l=30^{\circ}$. Their locations and inferred distance of $10 \mathrm{kpc}$ (based on hydrogen column density estimates) placed them within the " $5 \mathrm{kpc}$ arm," an inner spiral arm of the galaxy identified with radio, infrared, and CO molecular line observations (Hayakawa et al. 1977). GS 1843-02 was detected with a flux of 8 mcrab. Periodic pulses with frequency of $10.5432 \pm 0.0002 \mathrm{mHz}$ were detected, with a pulse profile having a deep narrow notch seen in all energy bands. A cross scan located the source to a $50^{\prime} \times 6^{\prime}$ error box $(90 \%$ confidence). The $S A S 3$ source 2S 1845-024 was within the original Ginga error box (Makino 1988) but is outside of the refined Ginga error box (Koyama et al. 1990b).

Koyama et al. (1990a) concluded that the six new sources they had discovered in the $5 \mathrm{kpc}$ arm were all transient accreting pulsars in Be star binary systems. Accreting pulsars in binary systems with Be (or Oe) stars form the largest class of known accreting pulsars. With the exception of X-Perseus, all of these systems are transients. Be stars are main-sequence stars of spectral type B that show Balmer

\footnotetext{
${ }^{1}$ Space Science Laboratory, NASA/Marshall Space Flight Center, ES 84, Huntsville, AL 35812; mark.finger@msfc.nasa.gov, scott@ gibson.msfc.nasa.gov, colleen.wilson@msfc.nasa.gov, robert.b.wilson@ msfc.nasa.gov, shuang.zhang@msfc.nasa.gov.

${ }^{2}$ Universities Space Research Association, 4950 Corporate Drive, Suite 100, Huntsville, AL 35806.

${ }^{3}$ Departments of Physics and Astronomy, 366 LeConte Hall, University of California, Berkeley, CA 94720; bildsten@fire.berkeley.edu.

${ }^{4}$ Department of Physics and Center for Space Research, Massachusetts Institute of Technology, Cambridge, MA 02139; deepto@space.mit.edu.

5 Space Radiation Laboratory, California Institute of Technology, Pasadena, CA 91125; prince@srl.caltech.edu.

${ }^{6}$ Department of Physics, University of Alabama in Huntsville, Huntsville, AL 35899.
}

emission lines (see Slettebak 1988 for a review). This line emission and a strong infrared excess is associated with the presence of a cool circumstellar envelope. There is strong observational evidence that this envelope has the form of a disk, extending along the Be star equatorial plane (e.g., Quirrenbach et al. 1997). In Be/neutron star binary systems the transient outbursts are thought to be fueled by accretion from this circumstellar envelope, frequently occurring near periastron passage. A review of $\mathrm{Be} /$ neutron star binary systems is given by Apparao (1994).

Zhang et al. (1996) analyzed data from the Compton Gamma Ray Observatory (CGRO) spacecraft's Burst and Transient Source Experiment (BATSE) for transient hard X-ray emission for the region of the sky near $l=30^{\circ}, b=0^{\circ}$. Using Earth occultation analysis techniques they identified six hard X-ray outbursts between 1991 April and 1995 February from a source, designated GRO J1849-03, which was localized to a $30^{\prime} \times 60^{\prime}$ error box $(90 \%)$ that contained both $2 \mathrm{~S} 1845-024$ and portions of the GS 1843-02 error box. The outbursts were regularly spaced by $241 \pm 1$ days, lasted about 13 days, and reached a peak flux of $75 \mathrm{mcrab}(20-100 \mathrm{keV})$. The outburst behavior suggested a $\mathrm{Be} /$ neutron star binary, with a natural candidate being GS $1843-02$. Pulsations from this source during the outbursts were searched for in the BATSE data but not detected at that time. Observations with the Beppo-SAX Wide-Field camera (Soffitta et al. 1998) during a predicted outburst of GRO J1849-03 in 1996 September identified this source with $2 \mathrm{~S} 1845-024$. Zhang et al. and Soffitta et al. suggested the identification of the source with the transient pulsar GS 1843-02. Pulsations were, however, not detectable in the Beppo-SAX data because of limited sensitivity, leaving this conjecture unconfirmed.

Here we present CGRO/BATSE observations of outbursts of pulsed flux from GS 1843-02, which are coincident with the outbursts of GRO J1849-03 = 2S 1845-024 detected in BATSE Earth occultation measurements. Observations of pulsations not detected by Zhang et al. (1996) were made possible by sensitivity enhancements 
resulting from improvements in BATSE pulsar analysis techniques (see $\S 2.1$ ). Pulse timing analyses of these observations are presented, which show that the outbursts occur near the periastron passage of a wide, highly eccentric orbit about a high-mass companion. In addition these observations show that the pulsar spins up during outbursts. We conclude from the measured spin-up rates and inferred mass accretion rates that an accretion disk is present during the outbursts. We suggest that the pulsar's orbit is inclined from its companion's equator, with an outburst initiated and an accretion disk formed during each passage of the pulsar through its companion's circumstellar envelope.

\section{OBSERVATIONS AND DATA ANALYSIS}

BATSE is an all-sky monitor designed to study gammaray bursts and transient source outbursts in the hard X-ray and soft gamma-ray bands (Fishman et al. 1989). The observations reported here use data from the eight Large Area Detectors (LADs), which are $2025 \mathrm{~cm}^{2}$ in area by $1.24 \mathrm{~cm}$ thick Na I(Tl) scintillators operated in the $20 \mathrm{keV}-1.8 \mathrm{MeV}$ band. These are located at the eight corners of the CGRO spacecraft. The LADs are uncollimated, with each detector viewing half of the sky. The large fields of view result in high background rates in the detectors, which are generally not easily modeled or subtracted. The flux of a discrete source can be sampled twice per spacecraft orbit by fitting the steps in count rates that occur when it rises or sets over the Earth's horizon (Earth occultation analysis; see Harmon et al. 1992). For pulsars with periods shorter than $\sim 500 \mathrm{~s}$, the variable portion of a pulsar's pulse profile can be detected by using Fourier analysis, epoch folding, or by fitting count rates over short intervals, using a model that includes a description of the pulse profile and a polynomial that accounts for the combined background and the average source flux (pulsar analysis; see Bildsten et al. 1997).

Our pulse timing analyses use the DISCLA channel 1 data, which consist of discriminator rates for the $20-50 \mathrm{keV}$ band, continuously read out from all eight detectors with a resolution of $1.024 \mathrm{~s}$. Results from Earth occultation analyses use the 16 energy channel CONT data, which are continuously read out from all eight detectors with a resolution of $2.048 \mathrm{~s}$.

\subsection{Blind Frequency Searches}

We have made blind searches for pulsations consistent with the $10.54 \mathrm{mHz}$ pulse frequency of GS 1843-02 (Koyama et al. 1990b) in the BATSE DISCLA data from 1991 July 16 to 1997 June 20. Zhang et al. (1996) searched the same data during the six outbursts of GRO J1849-03 they observed, resulting in no clear detection of pulses from GS $1843-02$. Through a combination of longer integration times, optimized combination of rates from different detectors, and improved handling of systematic effects due to interfering sources, our current searches are approximately 3 times more sensitive then those made by Zhang et al. (1996). With these improvements, and the extended search interval, we clearly detect 10 outbursts from GS $1843-02$, at times consistent with the outburst ephemeris of GRO J1849-03.

The data were divided into 4 day intervals, with each interval searched for pulsations in the barycentric frequency range of $10.47-10.61 \mathrm{mHz}$. The increase of the search interval from the 1 day used by Zhang et al. (1996) to 4 days provides a factor of 2 improvement of sensitivity for a con- stant frequency source. No additional increases in the search interval duration were made because of the increased danger that frequency changes due to intrinsic spin-up (or spin-down) and orbital motion would result in a loss of coherence (and hence sensitivity) in the search (Chakrabarty et al. 1997).

For each 4 day interval DISCLA channel 1 data were selected for which the source was visible, the high voltage was on, the spacecraft was outside of the South Atlantic Anomaly, and no electron precipitation events or other anomalies had been flagged by the BATSE mission operations personnel. The rates were combined over detectors using weights optimized for a source with an exponential spectrum $d N / d E=(A / E) \exp (-E / k T)$ with temperature $k T=25 \mathrm{keV}$ (see Bildsten et al. 1997, Appendix A). This weighting provides an improvement of sensitivity over the cosine of aspect angle weighting used by Zhang et al. (1996). These combined rates were grouped into segments of $\approx 300$ $\mathrm{s}$ duration. This segment duration was chosen because it contains several pulse periods and yet is short enough that a quadratic model generally provides a good fit to the detector background. Segment boundaries were chosen to avoid inclusion of occultation steps from the bright sources Cyg X-1, GRO J1744 - 28, and GRO J1655 - 40.

A pulse profile estimate was obtained from each segment by fitting the combined rates with a model composed of a sixth-order Fourier expansion in pulse phase (representing the pulse profile) combined with a quadratic in time (representing the background plus mean source rate). This resulted in an estimated pulse profile (represented by six Fourier coefficients) for each data segment. The phase model used in this initial fitting had a constant barycentric frequency of $v_{0}=10.54 \mathrm{mHz}$. Six harmonics were used so that the narrow notch seen in the Ginga pulse profiles could be reasonably represented.

The set of (typically several hundred) segment profiles for a given 4 day interval was then searched for a pulsed signal using a generalization of the $Z_{n}^{2}$ test of Buccheri et al. (1983). The $Z_{n}^{2}$ test assumes Poisson statistics. However, the noise in our data is often dominated by the aperiodic activity of Cyg X-1, which is usually in the field of view. Our test statistic, which we call the $Y_{n}$ statistic, accounts for this frequency-dependent excess noise.

We represent the pulse profile in the $m$ th $300 \mathrm{~s}$ data segment as

$$
r(t)=\mathfrak{R} \sum_{h=1}^{n} \alpha_{m h} \exp i 2 \pi h \phi_{0}(t),
$$

where $\mathfrak{R}$ denotes the real part, $n=6$ harmonics, $\alpha_{m h}$ is the estimated complex Fourier coefficient for interval $m$ and harmonic $h$, and the initial phase model is $\phi_{0}(t)=(t-\tau) v_{0}$, with $t$ the barycenter corrected observation time and the epoch $\tau=$ JD 245000.5 .

We fitted the segment profiles in a 4 day interval to a mean profile, which is represented as

$$
\bar{r}(\phi)=\mathfrak{R} \sum_{h=1}^{n} \mu_{h} \exp i 2 \pi h \phi,
$$

where $\mu_{h}$ are the estimated Fourier coefficients of the mean pulse profile. The pulse phase within segment $m$ is represented as

$$
\phi(t)=\phi_{0}(t)+\Delta \phi_{m},
$$


where

$$
\Delta \phi_{m}=\left(\bar{t}_{m}-\tau\right) \Delta v,
$$

with $\bar{t}_{m}$ the midpoint barycentric time of segment $m$ and $\Delta v$ a frequency correction estimated for the 4 day interval. Here we assume that the phase change within a $300 \mathrm{~s}$ segment caused by $\Delta v$ is a small fraction of a cycle.

The $\chi^{2}$ of the fit has the form

$$
\chi^{2}=\sum_{h=1}^{n} J_{h}\left(\mu_{h}\right)
$$

with

$$
J_{h}\left(\mu_{h}\right)=\sum_{m=1}^{M} \frac{\left|\alpha_{m h}-\mu_{h} \exp \left(i 2 \pi h \Delta \phi_{m}\right)\right|^{2}}{\sigma_{m h}^{2}},
$$

where $M$ is the number of $300 \mathrm{~s}$ segments in the 4 day interval and $\sigma_{m h}$ is the Poisson error of either the real or imaginary part of $\alpha_{m h}$.

The $Z_{n}^{2}$ statistic may be expressed as

$$
Z_{n}^{2} \equiv \sum_{h=1}^{n} \frac{\left|\mu_{h}^{\min }\right|^{2}}{\sigma_{\mu_{h}}^{2}}=\sum_{h=1}^{n}\left[J_{h}(0)-J_{h}^{\mathrm{min}}\right],
$$

where the $J_{h}^{\mathrm{min}}$ is the minimum of $J_{h}$ that occurs at the mean Fourier coefficient $\mu_{h}^{\min }$ and $\sigma_{\mu_{h}}$ is the formal (i.e., Poisson statistical) error of $\mu_{h}^{\min }$. To account for the non-Poisson errors, we multiply $\sigma_{\mu_{h}}^{2}$ by the reduced $\chi^{2}$ factor $J_{h}^{\min } /(2 M-2)$. This results in the following test statistic:

$$
Y_{n}=\sum_{h=1}^{n}\left[J_{h}(0)-J_{h}^{\min }\right] \frac{2 M-2}{J_{h}^{\min }} .
$$

For each 4 day search interval the $Y_{6}$ statistic was calculated for a grid of frequency offsets. The results are summarized in Figure 1. This shows for each search interval the peaks in $Y_{6}$ with a value above 35 , with the symbol size in proportion to $Y_{6}-35$. Monte Carlo calculations show that with no signal present we should expect $\approx 75$ peaks on the plot with $Y_{6}>35$ and a $50 \%$ chance of one peak with $Y_{6}>45$. We actually find 55 with $Y_{6}>45$ in Figure 1, with a maximum value of $Y_{6}=140$.

A series of 10 outbursts is clearly evident, as is a longterm spin-up trend in the pulse frequency of $\approx 2.7 \times 10^{-13}$ $\mathrm{Hz} \mathrm{s}^{-1}$. The arrows give the peaks of the outbursts of GRO J1849-03 detected by Zhang et al. (1996) using BATSE occultation techniques. These are consistent with the pulsed outbursts we have detected. The regular outbursting is typical of many of the Be star/pulsar systems observed by BATSE (Bildsten et al. 1997).

Figure 2 examines how the observed pulse frequency changes during the outbursts. For each 4 day interval we narrowed our frequency search range to a $14 \mu \mathrm{Hz}$ band centered on the linear frequency model $v_{\text {model }}(t)=v_{0}$ $+(t-\tau) \dot{v}$, with $v_{0}=10.585 \mathrm{mHz}, \tau=\mathrm{JD} 2450000.5$ and $\dot{v}=2.72 \times 10^{-13} \mathrm{~Hz} \mathrm{~s}^{-1}$. The frequencies of maximum $Y_{6}$ are plotted with this linear model subtracted if $Y_{6}>35$, with symbol areas proportional to $Y_{6}-35$. The abscissa is the observation times folded with a 242 day period, with epoch MJD 48362 (the first day of useful BATSE data). A 242 day period was used rather than the $241 \pm 1$ day orbital period estimate of Zhang et al. (1996) because it resulted in less scatter. The horizontal bars give the 4 day width of the search intervals. The rapid frequency decrease at the beginning of the outburst is undoubtedly due to the Doppler

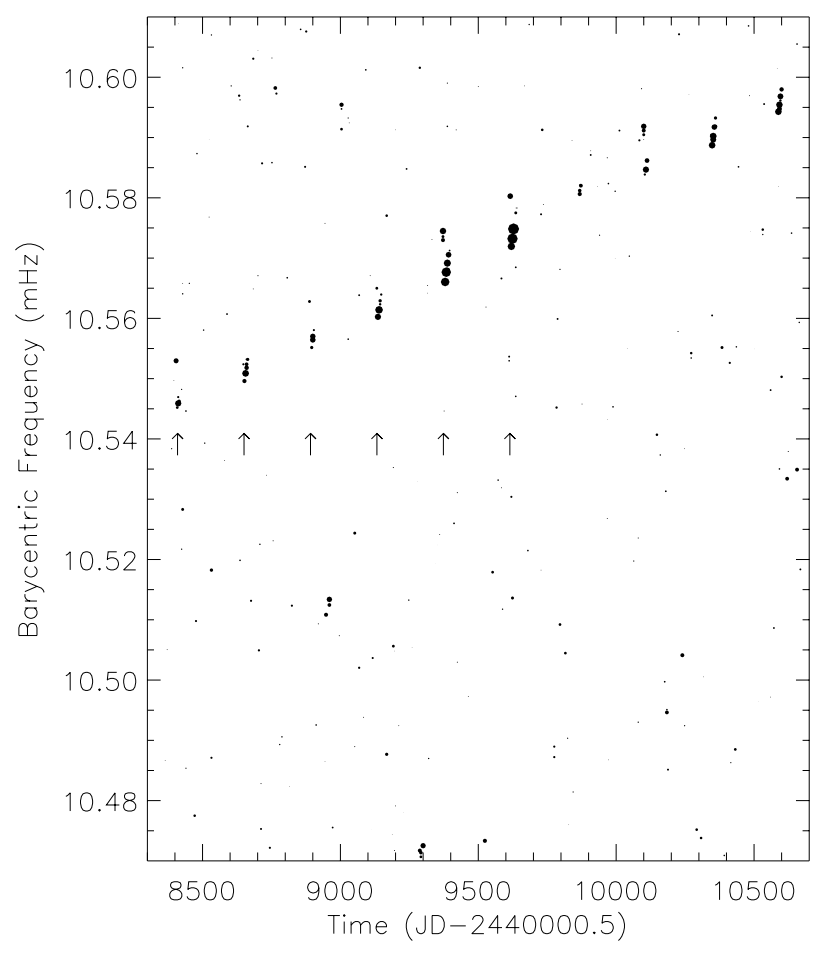

FIG. 1.-Blind search results for pulsations from GS 1843-02. For each 4 day search interval the plot shows peaks in the $Y_{6}$ statistic with value above 35 . The symbol area is proportional to $Y_{6}-35$. The arrows indicate the peak times of the outburst of GRO J1849-03 reported by Zhang et al. (1996). For reference JD 2448500.5 = 1991 September 1.0.

signature of an eccentric binary orbit. The slower increase in the observed frequency that follows could be due to both orbital Doppler shifts and intrinsic accretion-induced spin-up of the pulsar.

\subsection{Pulse Frequency Modeling}

To obtain a preliminary estimate of the system parameters and any intrinsic spin-up occurring during the outbursts, the frequencies in Figure 2 were fitted with a model that included an independent spin frequency and spin frequency rate for each outburst and a full set of binary orbital parameters. A new search of the 4 day intervals was then conducted, and new frequency estimates obtained. In this search the segment phase offsets in equation (4) were replaced with

$$
\Delta \phi_{m}=\phi_{\text {model }}\left(\bar{t}_{m}\right)+\Delta v\left(\bar{t}_{m}-\tau\right)-\phi_{0}\left(\bar{t}_{m}\right),
$$

where $\phi_{\text {model }}(t)$ incorporates the spin frequencies, frequency rates, and orbital parameters determined by the fit to the initial frequency estimates. This iterative step was needed to improve the sensitivity in intervals where rapid frequency changes were inferred, such as near the beginning of the outbursts. The search was restricted to frequency offsets $\Delta v$ less than $5.8 \mu \mathrm{Hz}$ from the new folding model. The model parameters were then reestimated using the barycentric frequencies determined for detections with $Y_{6}>35$.

The first column of Table 1 shows the estimated binary orbital parameters, while the spin-up rates during the outbursts, and those inferred between outbursts (using a 30 day outburst width) are given in Figure 3. The highly eccentric orbit has a mass function of $7 \pm 1 M_{\odot}$, suggesting a $9 M_{\odot}$ companion or larger. The outbursts occur close in phase to 


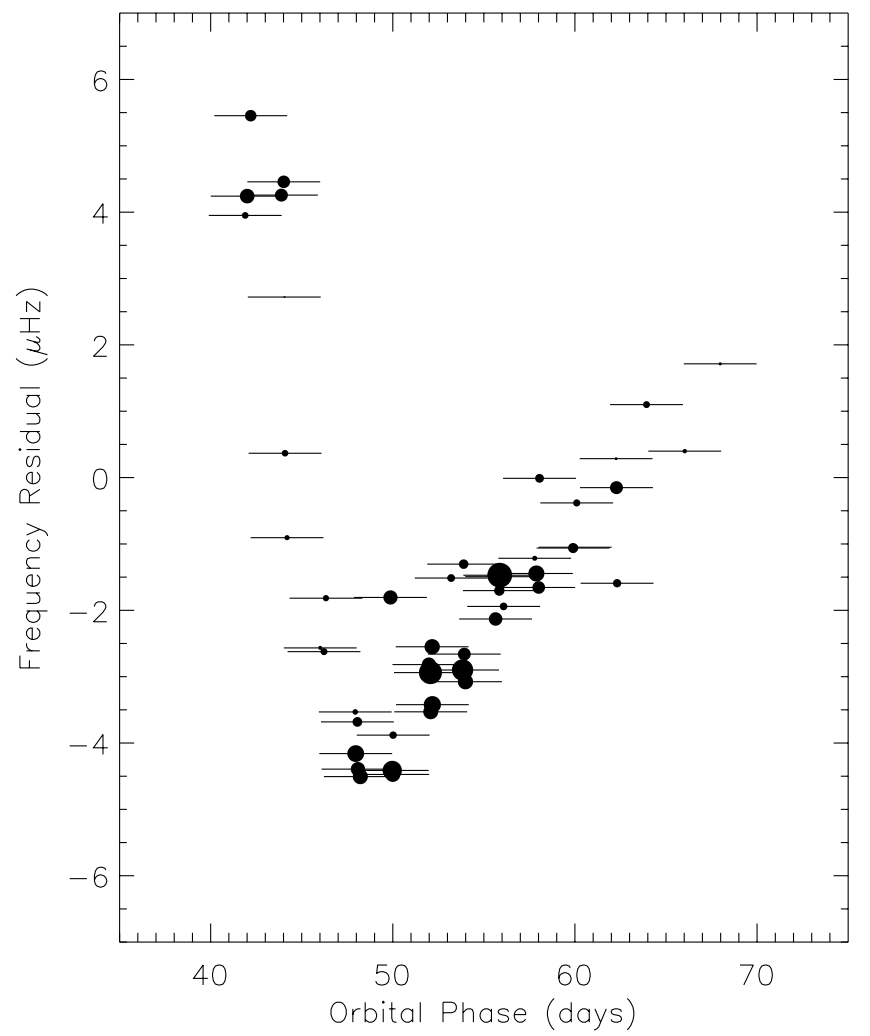

FIG. 2.-Frequency changes during the outbursts. Pulse frequencies were determined by searches over a narrow frequency band chosen to include the outbursts seen in Fig. 1. The detected frequencies minus a linear trend (see text) are plotted against the observation time folded at a 242 day period, beginning with MJD 48362, which provides an approximate orbital phase. A highly eccentric binary orbit combined with accretion induced spin-up caused the observed signature. The periastron epochs given in Table 1 are at phase 44 days in this plot.

the estimated periastron passage. The fit suggests that the long-term spin-up trend is the result of accretion torques that occur during the outbursts.

The quality of this fit is poor, with $\chi_{v}^{2}=34.3$. The fit residuals for most of the outbursts show significant structure, indicating that the simple constant frequency rate model used is inadequate. The errors given in Table 1 have been increased by the factor $\sqrt{\chi_{v}^{2}}$ to account for this poor fit, however, they are undoubtedly still underestimated. Adequate modeling will require parameters describing the time history of the torque during the outburst. These parameters will be strongly coupled to the orbital elements because of the limited orbital phase coverage. Such modeling will be studied in the pulse timing analysis that follows.

While statistically a poor fit, this model is sufficiently accurate for coherent epoch folding over intervals of a few

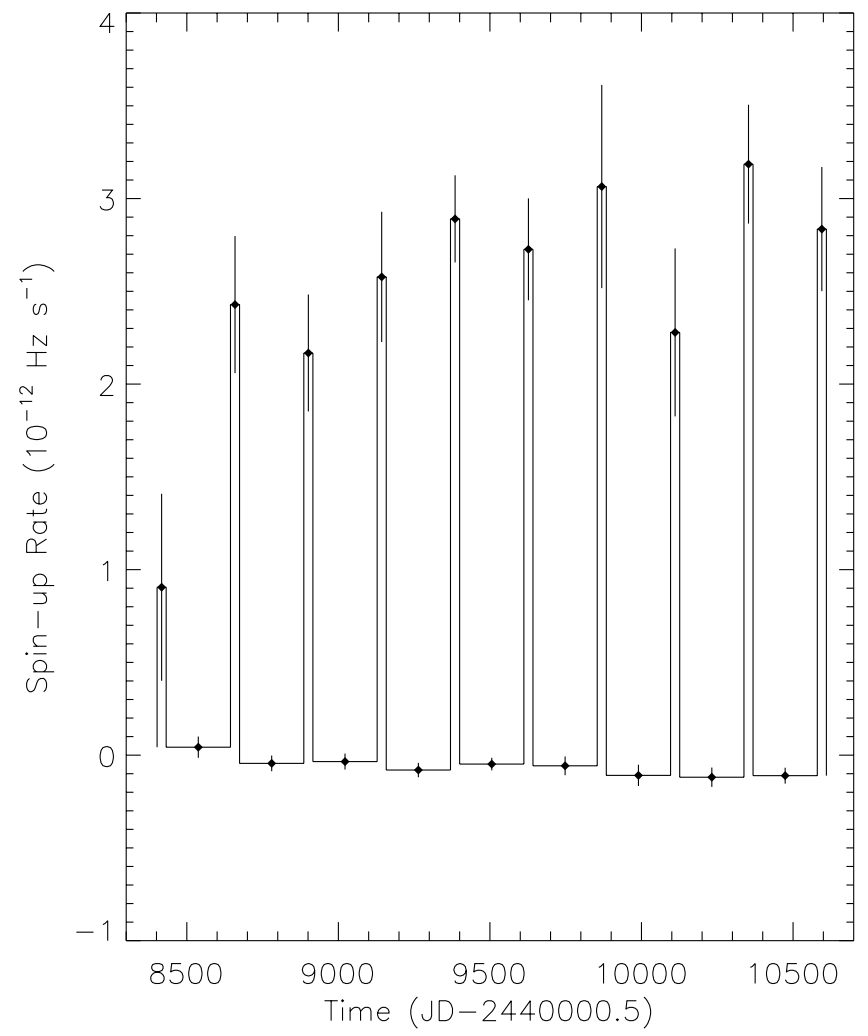

FIG. 3.-Intrinsic spin-up rates estimated by fitting the pulse frequencies. The model assumes that the spin-up rate is constant for 30 day intervals containing each outburst. The rate between outbursts is calculated from the model frequency difference between the edges of the adjacent outburst intervals.

days. The rms deviation of the measured frequencies from the model is $0.21 \mu \mathrm{Hz}$.

\subsection{Pulse Profile Measurements}

Figure 4 shows the mean pulse profile from the most significant 4 day interval (MJD 49624.0-49628.0). The profile is derived from estimates of its first six Fourier amplitudes, using the frequency model discussed in $\S 2.2$, and is therefore a smooth curve. Errors are shown at phases that are approximately statistically independent. The pulse amplitude is $\approx 20 \mathrm{mcrab} \mathrm{rms}(20-50 \mathrm{keV})$. This profile shows a narrow notch, which is similar to the GS 1843-02 profile observed by Ginga in the 9-38 keV range (Koyama et al. 1990b).

In preparation for a pulse timing analysis, one mean pulse profile was estimated every 2 days during the time range of each outburst for which pulsations were detected. Two profiles were made for each 4 day frequency search interval to provide higher resolution during the periastron

TABLE 1

Orbital Parameter Estimates

\begin{tabular}{ccc}
\hline Parameter & Frequency Fit & Phase Fit \\
\hline Period $\ldots \ldots \ldots \ldots \ldots \ldots \ldots \ldots$ & $242.18 \pm 0.02$ days & $242.180 \pm 0.012$ days \\
Periastron epoch $\ldots \ldots \ldots \ldots \ldots$ & JD $2449617.3 \pm 0.2$ & JD $2449616.98 \pm 0.18$ \\
Semimajor axis ............. & $725 \pm 40$ lt-s & $689 \pm 38 \mathrm{lt}-\mathrm{s}$ \\
Eccentricity $\ldots \ldots \ldots \ldots \ldots \ldots$. & $0.87 \pm 0.01$ & $0.8792 \pm 0.0054$ \\
Argument of periastron...... & $264^{\circ} \pm 7^{\circ}$ & $252^{\circ} .2 \pm 9.4$ \\
$\chi_{v}^{2} \ldots \ldots \ldots \ldots \ldots \ldots \ldots \ldots \ldots$. & $926 / 27$ & $55.1 / 52$ \\
\hline
\end{tabular}




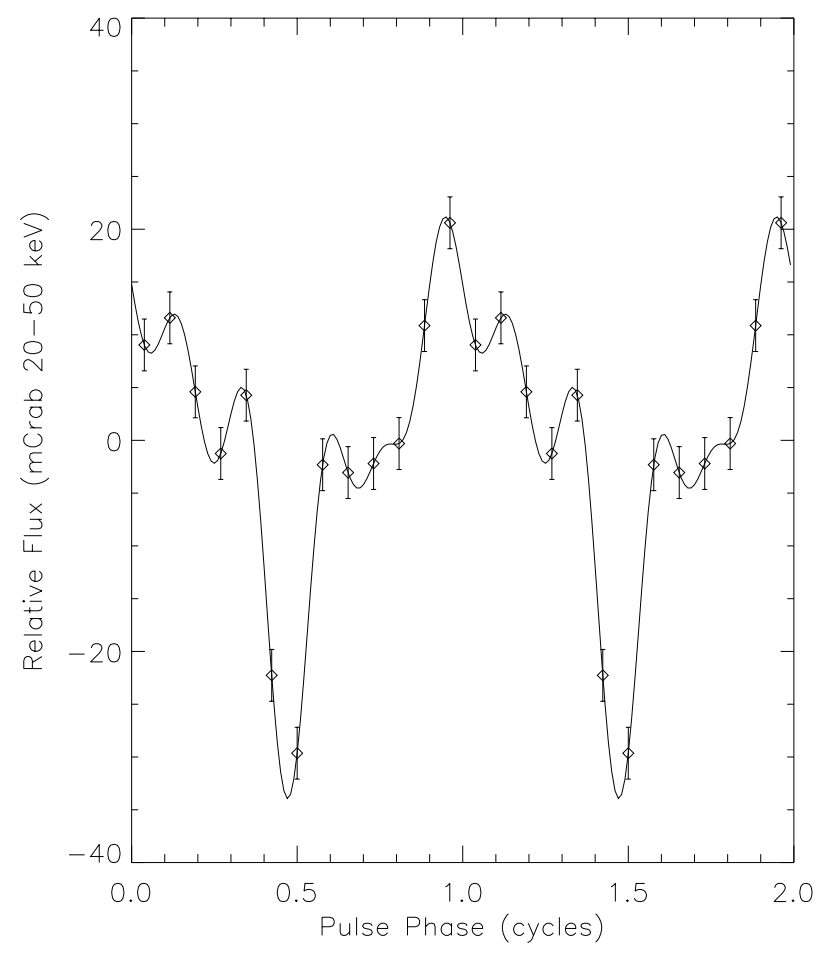

FIG. 4.-Pulse profile of GS $1843-02$ obtained from the BATSE 20-50 $\mathrm{keV}$ rates. The profile is limited to six harmonics. Error bars are give at approximately statistically independent points.

passage. We found that profiles from 1 day integrations were often not of sufficient significance to reliably estimate pulse phases. The first six Fourier amplitudes of each mean pulse profile were estimated from the $300 \mathrm{~s}$ profiles using the phase model obtained from the fit to pulse frequencies discussed above. The Poisson statistical errors of each Fourier amplitude $\mu_{k}$ were modified by the factor $\left[J_{h}\left(\mu_{h}\right) /(2 M\right.$ $-2)]^{1 / 2}$, where $M$ is the number of $300 \mathrm{~s}$ profiles, to account for non-Poisson noise. Several profiles from the edges of outbursts were dropped from further analysis because of low significance.

\subsection{Pulse Phase Measurements}

A phase offset $\Delta \phi$ from the phase model was estimated for each 2 day mean pulse profile by fitting the Fourier amplitudes $\mu_{h}$ of the profile to the Fourier amplitudes of a scaled and shifted template profile:

$$
\chi^{2}=\sum_{h=1}^{6} \frac{\left|\mu_{h}-A \exp (i 2 \pi \Delta \phi) T_{h}\right|^{2}}{\sigma_{\mu_{h}}^{2}} .
$$

Here $A$ is the estimated pulse amplitude, $T_{h}$ is a Fourier amplitude of the template profile, and $\sigma_{\mu_{h}}$ is the error on the real or imaginary component of $\mu_{h}$.

The template profile was obtained in three steps. Initially a cosine template was used to obtain phase offsets in the bright interval MJD 49620.0-49630.0. The profiles in this interval were then aligned using these offsets and a new template constructed from the weighted average of the aligned profiles. Using this template, phase offsets and amplitudes were than estimated for all the profiles. The 46 profiles with pulse amplitude above $5 \sigma$ significance were then aligned using these estimated offsets and the final template constructed from the weighted average of these aligned profiles.
For each 2 day interval, $j$, the mean observation time, reduced to the solar system barycenter, $t_{j}$, was computed. The total pulse phase at that time was then computed as $\phi_{j}=\phi_{\text {model }}\left(t_{j}\right)+\Delta \phi_{j}$, where $\Delta \phi_{j}$ is the estimated phase offset in interval $j$.

\subsection{Pulse Phase Modeling}

The pulse phases were fitted with a model that assumed a Gaussian profile for the spin-up rate during each outburst. In outburst $k$ the spin-up rate is described by three adjustable parameters; the peak spin-up rate $\dot{v}_{k}$, time of peak $t_{k}^{\text {peak }}$, and outburst width $W_{k}$. During outburst $k$ the fitted phase model had the form

$$
\begin{aligned}
\phi_{\text {model }}(t) & =\psi_{k}+v_{k}\left(t^{\mathrm{em}}-\tau_{k}\right) \\
+ & \dot{v}_{k}^{\text {peak }} \int_{t_{k} \text { peak }}^{\text {tem }} \int_{t_{k} \text { peak }}^{\hat{t}} \exp \left[-\frac{1}{2} \frac{\left(\tilde{t}-t_{k}^{\text {peak }}\right)^{2}}{W_{k}^{2}}\right] d \tilde{t} d \hat{t} .
\end{aligned}
$$

Here the phase constant $\psi_{k}$ and the frequency constant $v_{k}$ are adjustable parameters, while the epoch $\tau_{k}$ is fixed near the center of the outburst. The emission time $t^{\mathrm{em}}$ is related to the barycenter corrected observation time $t$ by

$$
\begin{aligned}
t=t^{\mathrm{em}}+a_{x} \sin i[\sin \omega( & \cos E-e) \\
& \left.+\sqrt{1-e^{2}} \cos \omega \sin E\right] / c,
\end{aligned}
$$

where

$$
E-e \sin E=\frac{2 \pi}{P_{\text {orbit }}}\left(t^{\mathrm{em}}-\tau_{\text {periastron }}\right),
$$

with the orbit's projected semimajor axis $a_{x} \sin i$, argument of periastron $\omega$, eccentricity $e$, orbital period $P_{\text {orbit }}$, and periastron epoch $\tau_{\text {periastron }}$ all being adjusted in the fit. The fitting was performed by minimizing

$$
\chi^{2}=\sum_{j=1}^{N} \frac{\left[\phi_{k}-\phi_{\text {model }}\left(t_{k}\right)\right]^{2}}{\sigma_{\phi_{k}}^{2}}
$$

using the Levenberg-Marquardt method (Press et al. 1992).

Initially only a single common value was estimated for the widths $W_{k}$ and the times of peak $\tau_{k}$ were constrained to be separated by the orbital period. This fit had a $\chi^{2}$ of 135.4 with 70 dof. Allowing the $\tau_{k}$ to be independently adjusted reduced the $\chi^{2}$ to 74.7 with 61 dof. With the widths $W_{k}$ also independently adjusted, $\chi^{2}$ dropped to 55.1 with 52 dof.

The orbital parameters obtained from this fit are shown in the second column of Table 1 . These are consistent with those obtained by fitting observed frequencies. The period, periastron epoch, and eccentricity are all well constrained by the pulse phase measurements. The argument of periastron $\omega$ and the semimajor axis $a_{x} \sin i$ are not as well constrained. Figure 5 shows their joint parameter confidence region. The broad minimum of $\chi^{2}$ for these two parameters is due to the difficulty in separating orbital and intrinsic torque signatures with data restricted to a small interval of orbital phase.

The estimated spin-up rate model is shown in Figure 6, which also shows the mean spin-up rate inferred between outbursts. Torques between outbursts contributed an estimated $5 \% \pm 23 \%$ of the long-term frequency change. The error on this estimate is coupled to those of $\omega$ and $a_{x} \sin i$, 


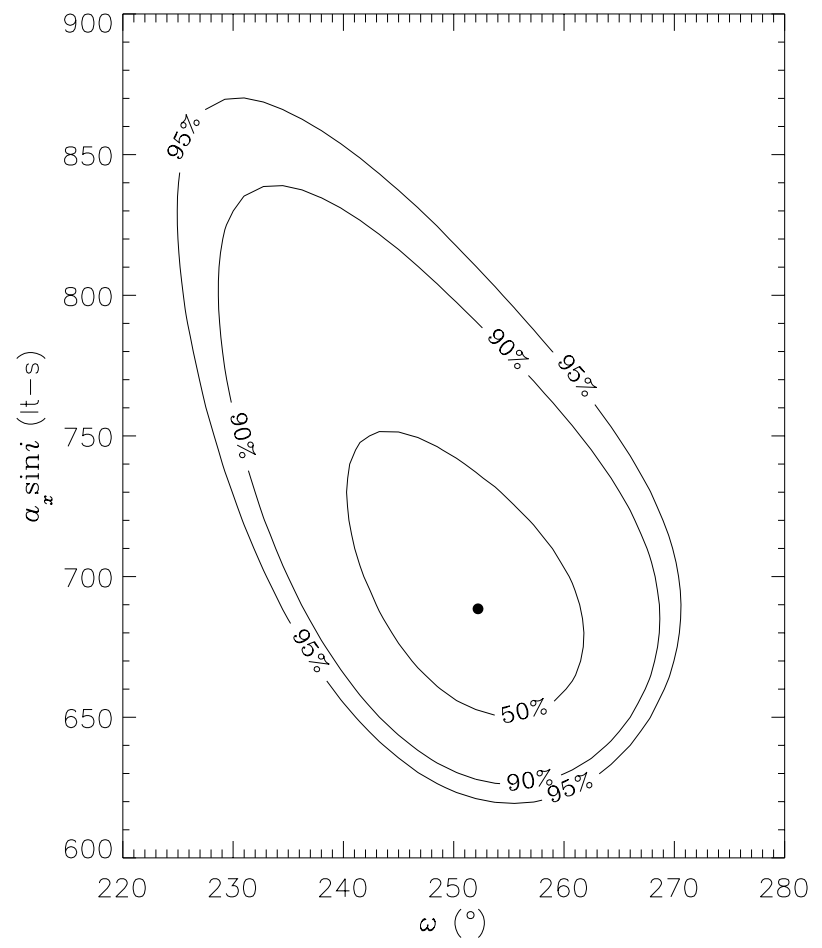

FIG. 5.- Joint confidence regions for the argument of periastron $\omega$ and the projected semimajor axis $a_{x} \sin i$ as estimated by fitting pulse phases.

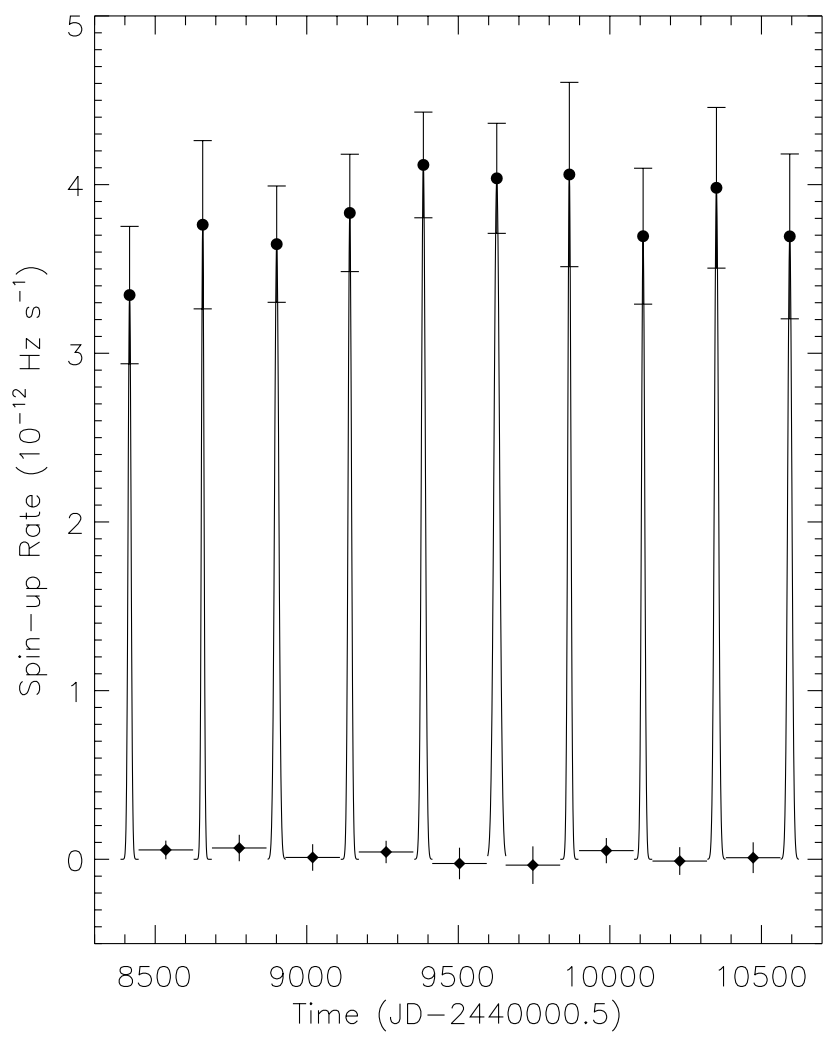

FIG. 6.- Intrinsic spin-up rates estimated by fitting the pulse phase. The model profile is Gaussian with adjustable width and peak time for each outburst. The solid circles give the peak spin-up rates. The average spin-up rates between outbursts are given by the solid diamonds. These are calculated from the estimated model for 182 day intervals centered between outbursts. with the estimated fraction varying from -0.33 to 0.26 for fits with fixed values of $\omega$ and $a_{x} \sin i$ within the $50 \%$ confidence contour shown in Figure 5.

Figure 7 shows the estimated delay of the spin-up rate peak from periastron passage. The mean delay is $9.4 \pm 0.7$ days, which is shown in the figure. The error on the mean delay is coupled with those of $\omega$ and $a_{x} \sin i$, with the estimated mean delay varying from 8.1 to 10.0 days for fits with fixed values of $\omega$ and $a_{x} \sin i$ within the $50 \%$ confidence contour shown in Figure 5. The errors on the individual delays are correlated because of this coupling between orbital and torque parameter errors. In order to show the significant differences between delays, the error shown for each delay is that of the difference of the delay from the mean delay.

Figure 8 shows the estimated width parameters $W_{k}$ of the spin-up rate profiles. This has a mean value of $6.2 \pm 1.0$ days, which is shown in the figure. The error on the mean width is coupled with those of $\omega$ and $a_{x} \sin i$, with the estimated mean varying from 5.2 to 7.6 days for fits with fixed values of $\omega$ and $a_{x} \sin i$ within the 50\% confidence contour shown in Figure 5. The errors on the individual widths are correlated because of this coupling between orbital and torque parameter errors. In order to show the significant differences between widths, the error shown for each width is that of the difference of the width from the mean width.

\subsection{Outburst Profile}

Figure 9 shows the light curve of the pulsed flux for the 1996 September outburst. The other outbursts are similar. A mean pulse profile was determined from 2 day intervals,

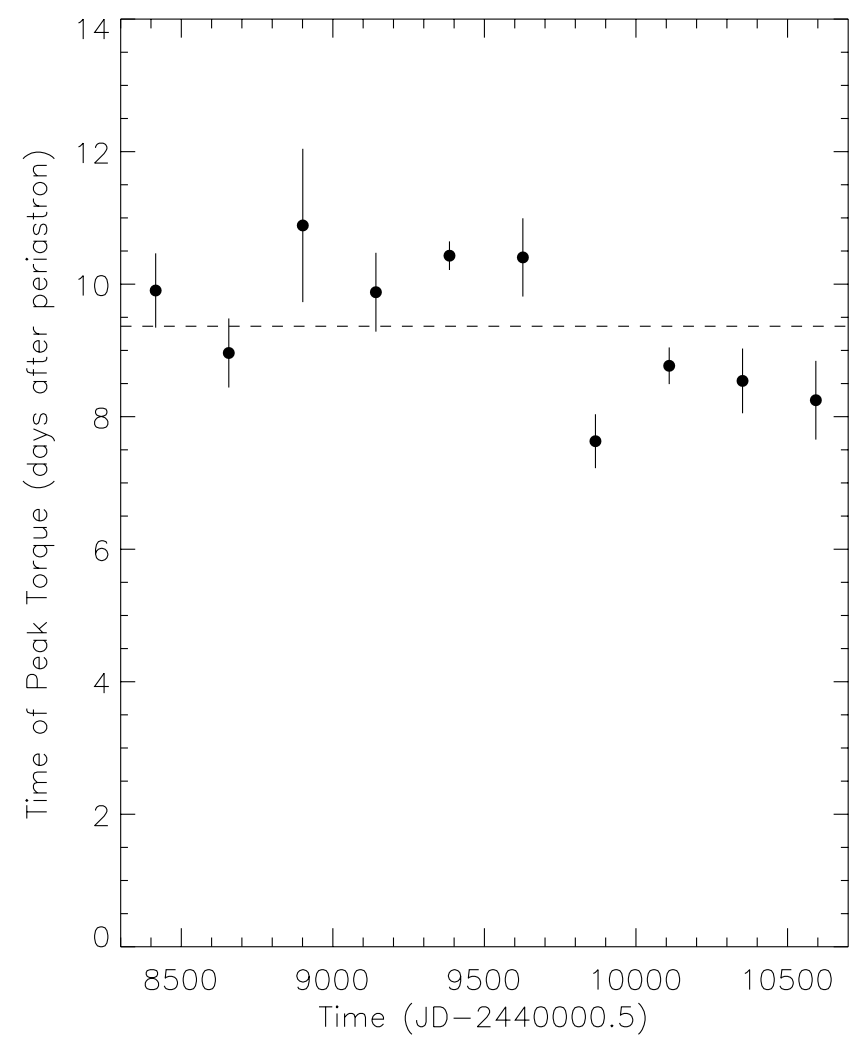

FIG. 7.- Times of peak spin-up rate $t_{k}^{\text {peak }}$ relative to periastron passage estimated from the pulse phase fit. The errors shown are for the difference from the mean delay of $9.4 \pm 0.7$ days, which is shown by the dashed line. 


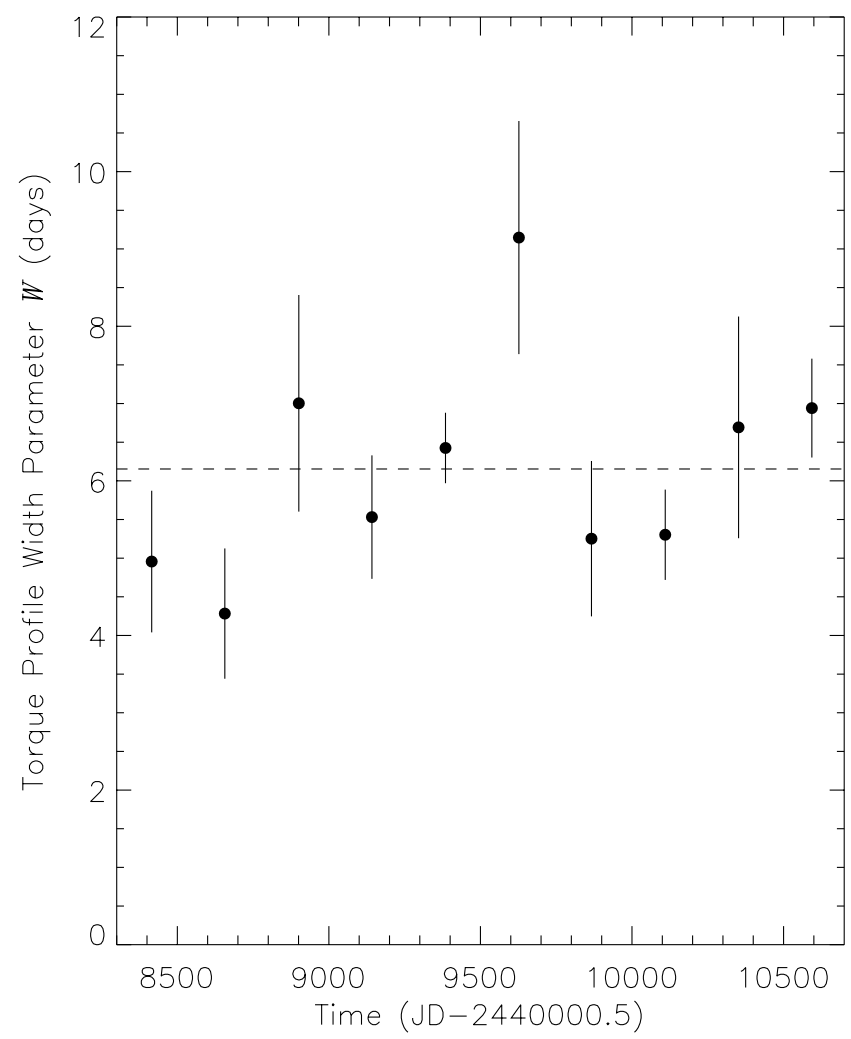

FIG. 8.-Estimated widths $W_{k}$ of the spin-up rate profiles. The errors shown are for the difference from the mean of $6.2 \pm 1.0$ days, which is shown by the dashed line.

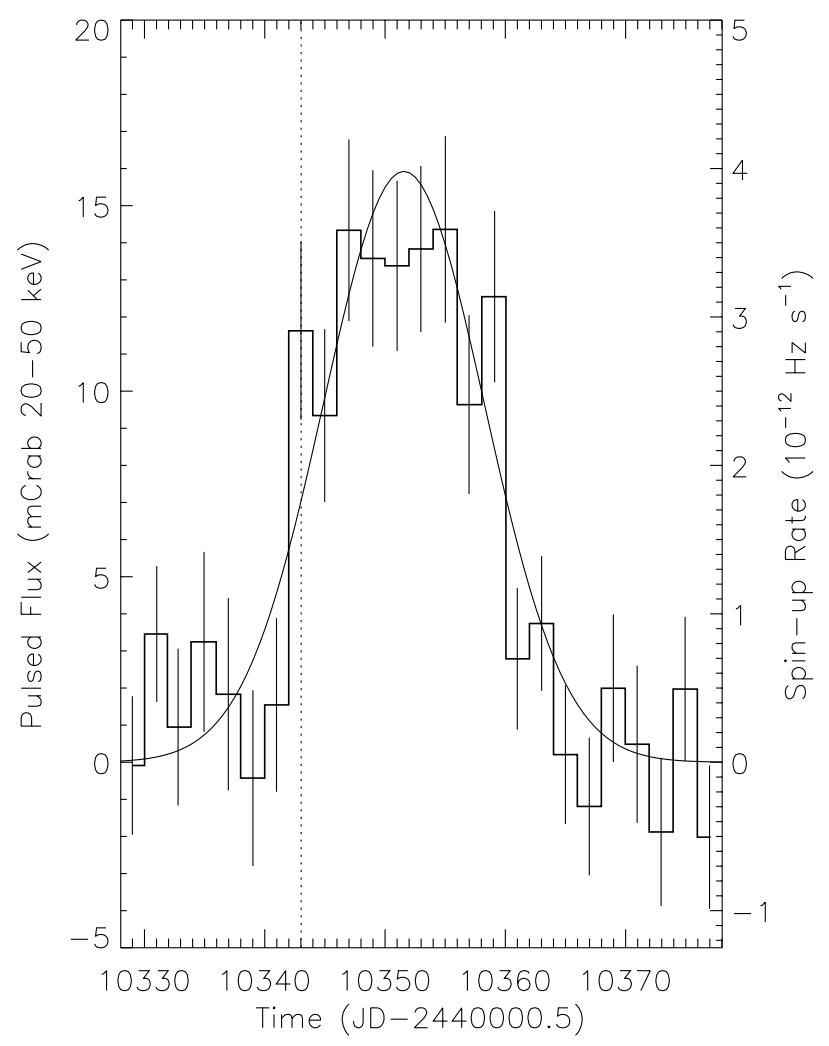

FIG. 9.-The $20-50 \mathrm{keV}$ pulsed flux (rms normalized) during the 1996 September outburst. Also shown is the model torque profile for this outburst (smooth curve). The dotted line is the time of periastron passage. using the detailed phase model estimated in the previous section to align the $300 \mathrm{~s}$ segment profiles. Non-Poisson noise was accounted for in the errors as previously described. The pulsed flux for each interval was calculated from the correlation of the mean pulse profile in that interval with the pulse template (with no adjustment in phase). The template was normalized to have unit variance, so that the correlation gives the rms pulsed flux if the pulse profile has the same shape as the template. Also shown in the plot is the model torque profile for the outburst and the time of periastron passage.

To examine the outburst light curve with higher significance, an average outburst profile was constructed. This is shown in Figure 10. The pulsed flux was determined as above for $P_{\text {orbit }} / 242 \approx 1$ day intervals and then epoch folded at the orbital period $P_{\text {orbit }}$. Also shown in the figure is the average model torque profile. Overall the pulsed flux and the torque profile are well aligned and of approximately the same width. Since the widths and peak times of the torque profile for each outburst were estimated in the phase modeling, this demonstrates a correlation between the spin-up rate and the pulsed flux.

There is a significant narrow feature in the average outburst light curve near periastron passage. This has a width of 2 days or less and is centered approximately a half day before periastron. Inspection of the 10 individual outburst light curves at 1 day resolution shows that this feature is present in the majority of them and is not caused by any individual flux enhancement.

In Figure 11 we show the average total flux profile obtained by epoch folding, at the orbital period, fluxes obtained by Earth occultation analysis (Harmon et al. 1992)

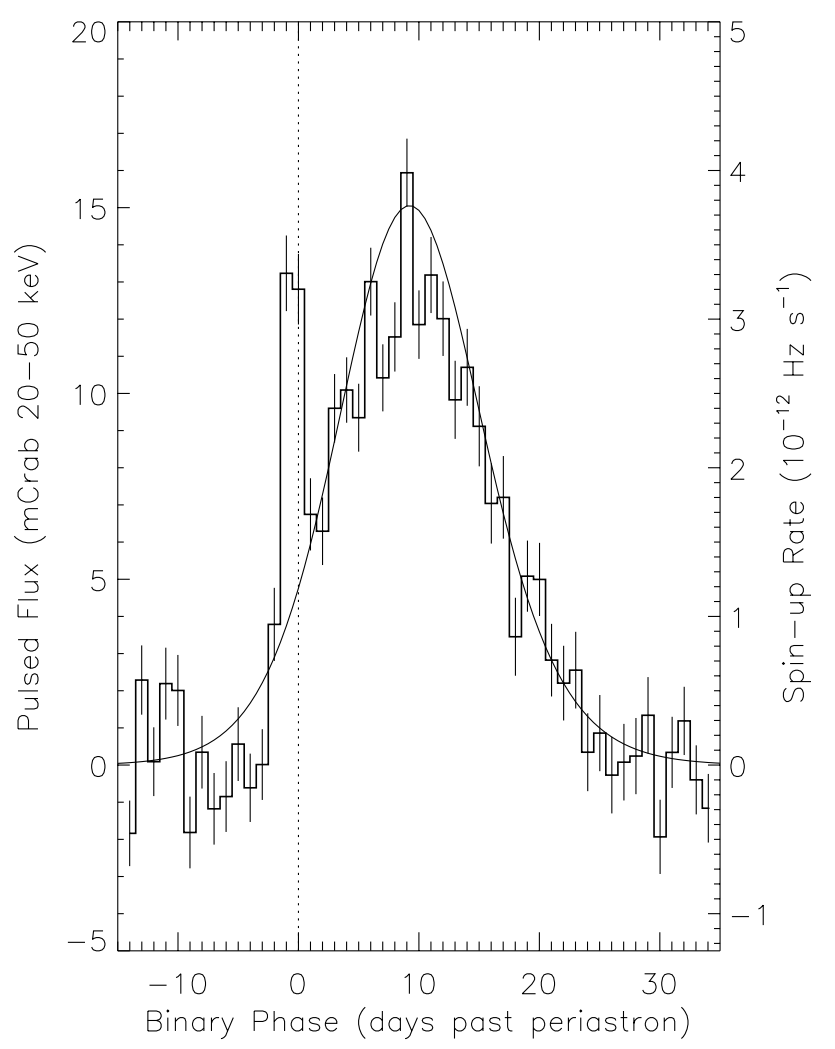

FIG. 10.-The $20-50 \mathrm{keV}$ pulsed flux (rms normalized) epoch folded at the orbital period. Also shown is the average model torque profile. 


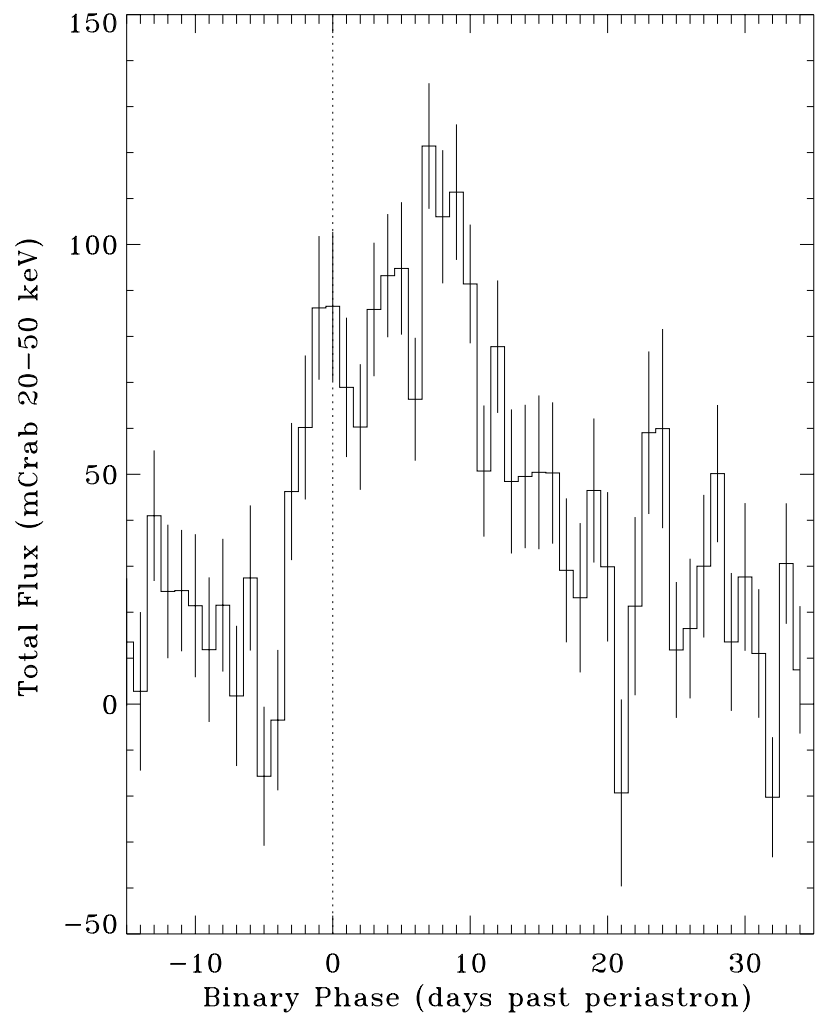

FIG. 11.-The 20-50 keV total flux (from BATSE Earth occultation measurements) epoch folded at the orbital period.

of the BATSE CONT data. This figure includes only the outbursts analyzed by Zhang et al. (1996) using data from MJD 48363-49741. The occultation fluxes were obtained from fits that used a power-law spectral model with a fixed photon number index of -2.5 . The onset time and width of the outburst roughly agree with those in Figure 10, but a detailed comparison is precluded by the higher relative noise level in the average total flux profile.

\section{DISCUSSION}

These observations show a series of 10 outbursts of the pulsar GS $1843-02$ occurring every 242 days. This outbursting behavior and the wide, highly eccentric orbit, which has a mass function requiring a companion mass of $M_{\text {opt }}>7 M_{\odot}$, leave little doubt that the source is a Be star/pulsar system. The consistency of the outburst intervals with those of the periodic transient GRO J1849-03 (Zhang et al. 1996) clearly shows these to be the same source. Beppo-SAX Wide-Field Camera observations of GRO 1849-03 during a predicted outburst in 1996 September have recently identified this source with $2 S$ 1845-024 (Soffitta et al. 1998); 2S 1845-024 was observed by SAS 3 in 1975 July and September and localized to 35" (Doxsey et al. 1977). As shown by Soffitta et al. (1998) the recent outbursts were also seen in the Rossi X-Ray Timing Explorer (RXTE) All Sky Monitor (ASM) light curve of $2 \mathrm{~S}$ $1845-024$.

Other observations of outbursts and additional identifications might possibly be found by examining sources cataloged in the region of $2 \mathrm{~S} 1845-024$ by early missions. This includes A1845-02 and H1845-024. A1845-02 was discovered by Ariel 5 in 1974 November (Villa et al. 1976) and identified with $2 \mathrm{~S} 1845-024$ by Doxsey et al. (1977). H1845-024 is listed in the HEAO 1 catalog (Wood et al. 1984) with the assumed location of $2 S 1845-024$. In Table 2 we list observations of $2 \mathrm{~S} 1845-024$ and its possible counterparts along with the calculated orbit phase during the observations. The detections by Ariel 5, SAS 3, Ginga, Beppo-SAX, and RXTE are all consistent with the range of orbital phases in which BATSE detects pulsations, which is from 4 days before to 24 days after periastron passage. The $H E A O$ observation, however, occurs near apastron. This detection is possibly due to source confusion with another transient. Several other transient pulsars have been found in this region (Koyama et al. 1990a) since the HEAO 1 catalog analysis.

BATSE observations of accreting pulsars (Bildsten et al. 1997) have shown that extended series of low-luminosity $\left(10^{36}-10^{37}\right.$ ergs $\left.\mathrm{s}^{-1}\right)$ outbursts occurring near periastron are common in the transient Be star/pulsar systems. The outbursts of $2 \mathrm{~S} 1845-024$ observed by BATSE fit into this "normal" outbursting pattern. The peak luminosity determined for the 1996 September outburst from combined RXTE ASM (2-12 keV) and BATSE occultation data $(20-100 \mathrm{keV})$ was $6 \times 10^{36} \mathrm{ergs} \mathrm{s}^{-1}$, assuming a distance of $10 \mathrm{kpc}$ (Soffitta et al. 1998). As shown in Table 2 the peak fluxes of the outbursts observed by BATSE are all fairly similar. The outbursts have proceeded like clockwork since at least the launch of the CGRO. Figure 12 shows the Ginga and BATSE frequency measurements, corrected for the orbit in the second column of Table 1. Significant spin-up occurred between the Ginga and the BATSE measurements, but that spin-up was smaller than would be predicted from an extrapolation of the BATSE measurements. If, as suggested by our estimates, the long-term spin-up is due largely to torques occurring within outbursts, then unobserved

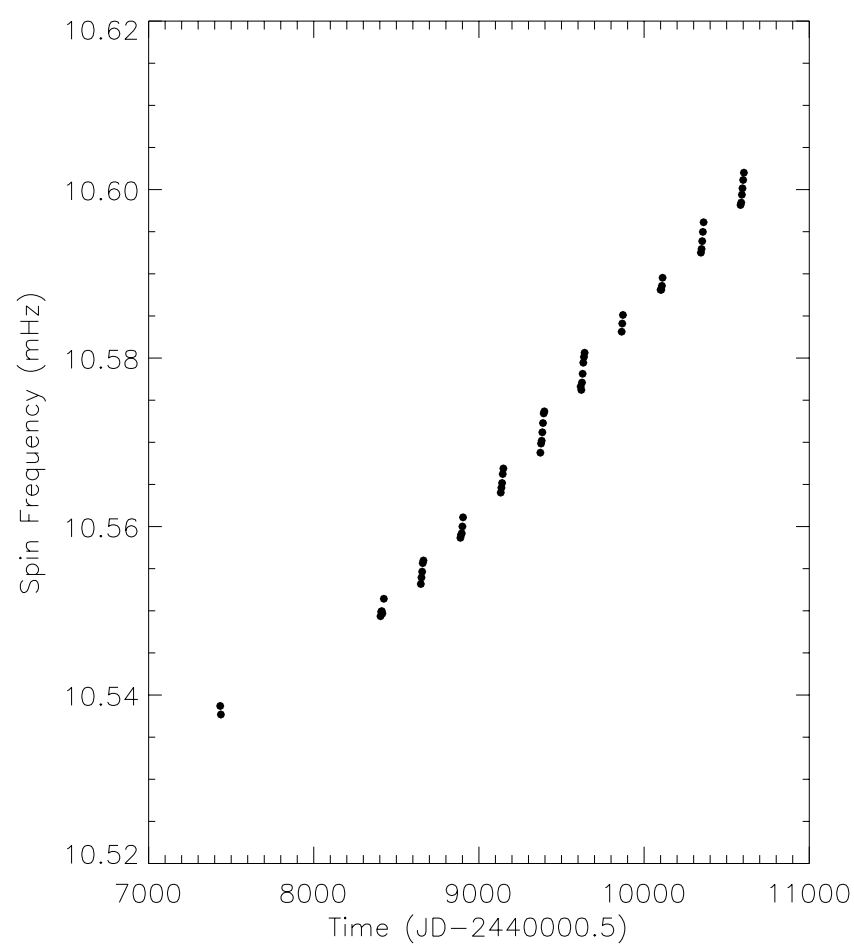

FIG. 12.- Long-term history of the spin frequency, corrected using the orbit in Table 2. The initial points are from the Ginga pointed observations, the remainder from BATSE observations. 
TABLE 2

Orbital Phase of Observations

\begin{tabular}{|c|c|c|}
\hline Date & $\begin{array}{c}\text { Phase } \\
\text { (Days past Periastron) }\end{array}$ & $\begin{array}{l}\text { Peak Flux } \\
\text { (mcrab) }\end{array}$ \\
\hline \multicolumn{3}{|l|}{ Ariel 5 $5^{\text {a }}(2-6 \mathrm{keV})$ : } \\
\hline 1974 Nov $12-25$............ & $11-26$ & 12 \\
\hline 1975 Jul 21-Aug 16 ........ & $21-48$ & 2 \\
\hline \multicolumn{3}{|l|}{$S A S 3^{\mathrm{b}}(2-11 \mathrm{keV})$} \\
\hline 1975 Jul $11-16 \ldots \ldots \ldots \ldots \ldots$ & $11-17$ & 9 \\
\hline \multicolumn{3}{|l|}{ HEAO $1^{\mathrm{c}}(2-10 \mathrm{keV})$} \\
\hline 1977 Oct $2-10 \ldots \ldots \ldots$ & 98-106 & 7 \\
\hline \multicolumn{3}{|l|}{ Ginga $\operatorname{LAC}^{\mathrm{d}}(2-20 \mathrm{keV})$ : } \\
\hline 1987 Oct 6 ............... & -120 & $<0.25$ \\
\hline 1987 Oct $10 \ldots \ldots \ldots \ldots \ldots$ & -116 & $<0.25$ \\
\hline 1988 Sep $12 \ldots \ldots \ldots \ldots \ldots$ & -20 & $<0.25$ \\
\hline $1988 \operatorname{Sep} 29 \ldots \ldots \ldots \ldots \ldots$ & -3 & 8 \\
\hline 1988 Oct $3 \ldots \ldots \ldots \ldots \ldots$ & 1 & 9 \\
\hline \multicolumn{3}{|l|}{ BATSE (pulsed, 20-50 keV): } \\
\hline 1991 May 28-Jun $16 \ldots \ldots$. & $-2-18$ & $18 \pm 5$ \\
\hline 1992 Jan $25-$ Feb $13 \ldots \ldots \ldots$ & $-2-18$ & $12 \pm 2$ \\
\hline 1992 Sep $21-$ Oct $12 \ldots \ldots \ldots$ & $-4-18$ & $14 \pm 2$ \\
\hline 1993 May $23-$ Jun $11 \ldots \ldots$. & $-2-18$ & $15 \pm 2$ \\
\hline 1994 Jan $18-F e b 14 \ldots \ldots \ldots$ & $-4-24$ & $15 \pm 2$ \\
\hline 1994 Sep $21-$ Oct $12 \ldots \ldots \ldots$ & $-1-22$ & $14 \pm 2$ \\
\hline 1995 May $19-$ Jun 7 ........ & $-3-17$ & $20 \pm 4$ \\
\hline 1996 Jan $16-F e b 8 \ldots \ldots \ldots$ & $-3-21$ & $13 \pm 2$ \\
\hline 1996 Sep $16-$ Oct $5 \ldots \ldots \ldots$ & $-1-19$ & $14 \pm 3$ \\
\hline 1997 May $14-J u n ~ 8 \ldots \ldots \ldots$. & $-3-23$ & $15 \pm 2$ \\
\hline \multicolumn{3}{|c|}{ Beppo-SAX Wide-Field Camera ${ }^{\mathrm{e}}(5-21 \mathrm{keV})$ : } \\
\hline 1996 Sep $17-18 \ldots \ldots \ldots \ldots . .$. & $0-1$ & 9 \\
\hline \multicolumn{3}{|l|}{$R X T E \operatorname{ASM}^{\mathrm{f}}(2-12 \mathrm{keV}):$} \\
\hline 1996 Sep $22-$ Oct $3 \ldots \ldots \ldots$ & $5-17$ & $21 \pm 2$ \\
\hline 1997 May $20-$ May $27 \ldots \ldots$. & $3-11$ & $22 \pm 2$ \\
\hline
\end{tabular}

${ }^{\text {a }}$ A 1845-02 was detected in scans folded over the given time ranges (Villa et al. 1976). Fluxes were calculated from the high-energy system count rates. Cross scans are mentioned, but accurate dates are not given.

${ }^{\mathrm{b}}$ From Doxsey et al. 1977.

${ }^{c}$ From Wood et al. 1984. The observation interval was calculated from the ecliptic longitude of 2S $1845-024$.

${ }^{d}$ From Koyama et al. $1990 \mathrm{~b}$. The fluxes were calculated from the $2-20$ $\mathrm{keV}$ count rates.

${ }^{\text {e }}$ From Soffitta et al. 1998.

${ }^{\mathrm{f}}$ From 4 day averages using $1 \mathrm{crab}=75 c \mathrm{~s}^{-1}$.

outbursts must have occurred between the Ginga and BATSE observations. The lower spin-up rate implies that these outbursts were on average smaller or that one or more periastron passages occurred without an outburst.

The BATSE observations of $2 \mathrm{~S} 1845-024$ provide the best evidence to date of spin-up occurring during normal outbursts of a transient pulsar. Spin-up during the less common "giant" outbursts of transient pulsars has been reported many times. During giant outbursts, which peak near the Eddington luminosity, an accretion disk is known to be present (Finger, Wilson, \& Harmon 1996b). Only recently, however, has evidence for spin-up during normal outbursts emerged with BATSE observations of $2 \mathrm{~S}$ 1417-624 (Finger, Wilson, \& Chakrabarty 1996a), GS 0834-430 (Wilson et al. 1997), and EXO 2030+375 (Stollberg et al. 1999) and now the observations of $2 \mathrm{~S}$ 1845-024 present here. The most common explanation for normal outbursting activity in transient Be star/pulsar systems is the direct accretion of the dense equatorial wind (or circumstellar disk) of the Be star (Waters et al. 1989) as the neutron star passes through periastron. Yet wind accretion is thought to be very inefficient at transferring angular momentum (Ruffert 1997), leading us to expect any spin-up during normal outbursts to be fairly small.

The spin-up rate we see during outbursts of $2 \mathrm{~S} 1845-024$ and its correlation with the pulsed flux (Fig. 10) suggests that an accretion disk is present. A disk will form if the specific angular momentum $l$ in a wind exceeds the Keplerian specific angular momentum $l_{m}=\left(G M r_{m}\right)^{1 / 2}$ at the magnetospheric radius $r_{m}$. After an accretion disk is formed, the specific angular momentum of accreting material is maintained near $l_{m}$. For accretion to occur, the magnetospheric radius must be within the corotation radius $r_{\mathrm{co}}=(G M)^{1 / 3}(2 \pi v)^{-2 / 3}$. Therefore, the specific angular momentum needed for disk formation, $l_{m}$, is bound by

$$
l_{m}<l_{\mathrm{co}}=\left(G M r_{\mathrm{co}}\right)^{1 / 2}=8 \times 10^{17} \mathrm{~cm}^{2} \mathrm{~s}^{-1} .
$$

From the luminosity and spin-up rate at the peak of the 1996 September outburst (which is typical) we infer a specific angular momentum of the accreting material of

$$
l=2 \pi I \dot{v} \dot{M}^{-1}=8 \times 10^{17} d_{10}^{-2} \mathrm{~cm}^{2} \mathrm{~s}^{-1}
$$

assuming $\dot{M}=L(G M / R)^{-1}$. Here $\dot{v}=4 \times 10^{-12} \mathrm{~Hz} \mathrm{~s}^{-1}$ is the spin-up rate, $\dot{M}$ is the mass accretion rate, $L=6 \times 10^{36}$ 
ergs $\mathrm{s}^{-1} d_{10}^{2}$ is the luminosity, and $d_{10}$ is the distance in units of $10 \mathrm{kpc}$. We have assumed $I=10^{45} \mathrm{~g} \mathrm{~cm}^{2}$ for the neutron star moment of inertia, $M=1.4 M_{\odot}$ for the mass, and $R=10^{6} \mathrm{~cm}$ for the radius. From comparison of equations (15) and (16) we conclude that disk accretion is occurring during the outbursts of $2 S$ 1845-024. By moving the source farther away we could avoid this conclusion. The distance of $10 \mathrm{kpc}$ estimated by Koyama et al. (1990b) was, however, based on the minimum column density determined for a set of Ginga spectra. Since part of this column density may be intrinsic to the source, a closer distance is more likely than a farther one.

The progenitor system of a Be/neutron star binary is likely a compact binary system of B stars (van den Heuvel 1994). The more massive star evolves more rapidly and transfers mass to its companion via Roche lobe overflow, resulting in a helium star in a circular orbit about its massenhanced companion. The helium star then undergoes a supernova explosion producing a neutron star in a wide eccentric orbit, if the system remains bound. Because the kick velocities produced by the supernova are thought to be larger than or comparable to typical orbital velocities of the presupernova systems, many such systems are expected to be disrupted.

Figure 13 shows the kick velocity required for various possible progenitor systems to have produced the $2 \mathrm{~S}$ 1845-024 system (see, e.g., Kalogera et al. 1996 for the relevant equations). Before the supernova the orbital angular momentum and the companion's spin angular momentum are aligned, while afterward they are separated

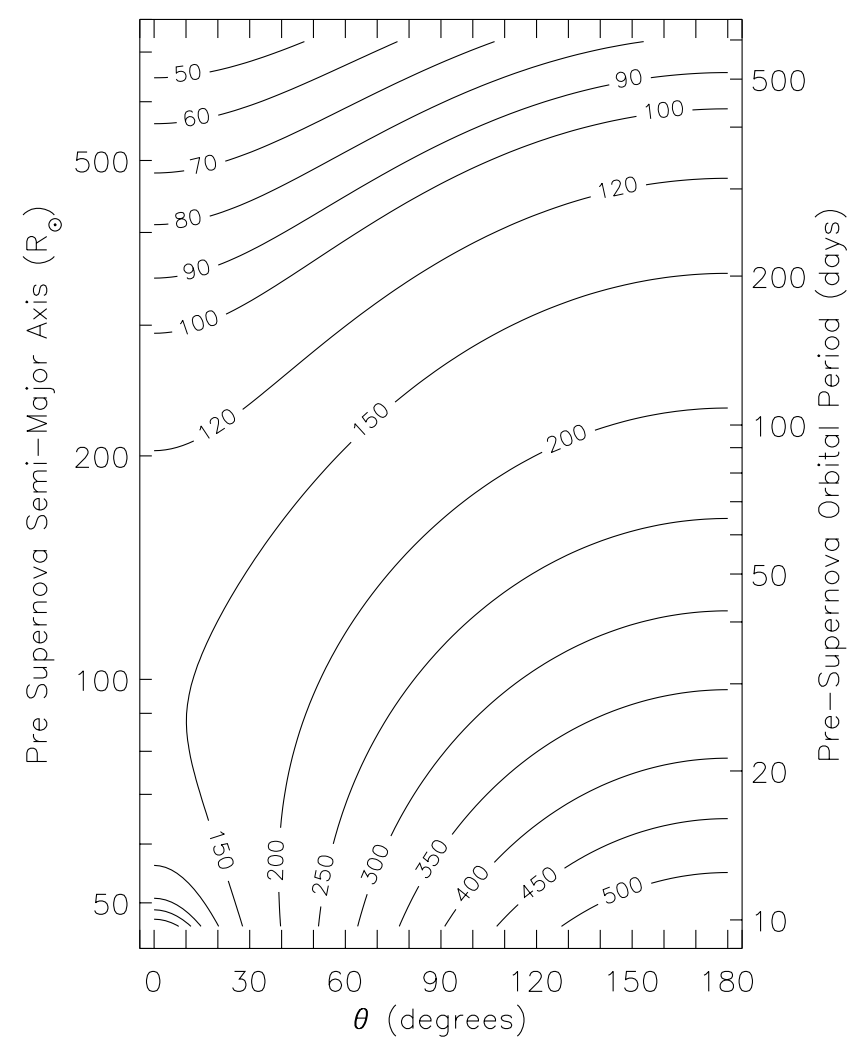

FIG. 13.-Kick velocity $\left(\mathrm{km} \mathrm{s}^{-1}\right)$ required to produce the present orbit for presupernova systems with a companion mass of $11.3 M_{\odot}$, and a neutron star mass of $1.4 M_{\odot} ; \theta$ is the angle between the orbital angular momentum and the Be star spin angular momentum after the supernova explosion. by the angle $\theta$. The calculation assumes a neutron star mass of $1.4 M_{\odot}$, a presupernova helium star mass of $3.0 M_{\odot}$, and a companion star mass of $11.3 M_{\odot}\left(i=60^{\circ}\right)$. The current eccentricity, period, and semimajor axis are assumed unchanged since the supernova. These elements are unaffected by the tidal interactions and spin-orbital coupling, which lead to periastron advance and precession of the orbital plane and the Be star spin axis (Lai, Bildsten, \& Kaspi 1995). For these parameters a minimum kick of 41 $\mathrm{km} \mathrm{s}^{-1}$ is required, which occurs in a 595 day period system with kick directed along the direction of motion. On the other hand, a kick of $550 \mathrm{~km} \mathrm{~s}^{-1}$ directed opposite to the direction of motion in a 9.7 day period system could have produced the system. In this case the $\mathrm{Be}$ star spin and orbital angular momentum are in opposite directions. The actual origin of the current system probably lies between these extremes. In particular we wish to emphasis that if typical kick velocities are $\sim 250 \mathrm{~km} \mathrm{~s}^{-1}$ (Hansen \& Phinney 1997), then there is no reason to suppose that the orbital plane and the $\mathrm{Be}$ star equator are closely aligned, as is normally assumed in the direct wind accretion outburst model (Waters et al. 1989).

We propose that the pulsar's orbit is inclined to its companion's equator and that the narrow feature in the outburst profile (Fig. 10) near the onset of the outburst is due to direct wind accretion during the pulsar's passage through the companion's circumstellar envelope. Figure 14 shows the pulsars separation from its companion, and its true anomaly, for times close to periastron. The geometry is changing rapidly, with the separation changing by a factor of 2 in less than 4 days and the true anomaly changing by $43^{\circ}$ day $^{-1}$ at periastron. If the angle between periastron and

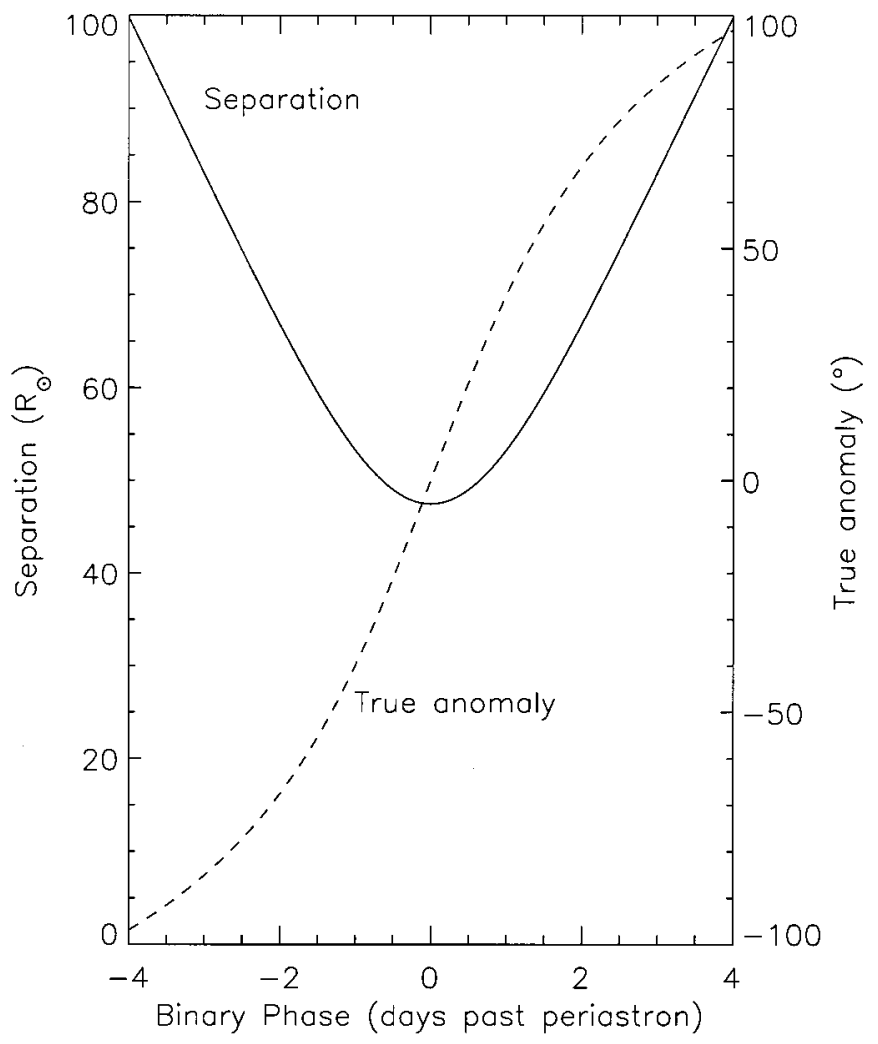

FIG. 14.-Neutron star's separation from its companion and the true anomaly, for times near periastron passage. The separation is calculated assuming a neutron star mass of $1.4 \mathrm{M}_{\odot}$ and an inclination of $60^{\circ}$. 
the node of the orbit with the companion's equator is less than $40^{\circ}$, then passage through the equatorial plane will occur within a day of periastron passage. Be star circumstellar envelopes have half-angles of less than $10^{\circ}$ (see Hanuschik 1996; Quirrenbach et al. 1997; Wood, Bjorkman, \& Bjorkman 1997). The passage through the circumstellar envelope would therefore take less than a day for orbits inclined from the companion's equator by more than $15^{\circ}$. Direct wind accretion during the passage could result in the onset feature in the profile. At this time the accretion disk could also be forming. Alternatively a disk could already exist (Bildsten et al. 1997), with enhanced accretion initiated near periastron because of tidal torques and newly added material.

This neutron star's wide and eccentric orbit about a massive star is similar to two radio pulsars PSR B1259-63 (Johnston et al. 1992) and PSR J0045-07319 (Kaspi et al. 1994). Both of these pulsars are in highly eccentric orbits $(e=0.8698,0.8080)$ about $8-10 M_{\odot}$ main-sequence stars with periods of 1236 and 51.2 days, respectively. Bildsten et al. (1997) had earlier noted the lack of accreting pulsars with similarly large eccentricities and conjectured that it was a selection effect due to the difficulties of repeatedly observing long-period systems. $2 \mathrm{~S} 1845-024$ begins to fill in this gap. As we have suggested for 2S 1845-024, the orbital plane of PSR J0045 - 7319 is believed to be inclinated from its companion's equator (Lai, Bildsten, \& Kaspi 1995; Kaspi et al. 1996). However, given its low spin frequency, it is unlikely that $2 \mathrm{~S} 1845-024$ would be a radio pulsar now or in the future.

M. H. F. acknowledges support from NASA grant NAG5-4238. L. B. and T. A. P. acknowledge support from NASA grant NAGW-4517. We thank the anonymous referee for helpful comments, which have improved the text.
Apparao, K. M. V. 1994, Space Sci. Rev., 69, 255

Bildsten, L., et al. 1997, ApJS, 113, 367

Buccheri, R., et al. 1983, A\&A, 128, 245

Chakrabarty, D., et al. 1997, ApJ, 474, 414

Doxsey, R. E., Apparao, K. M. V., Brandt, H. V., Dower, R. G., \& Jernigan, J. G. 1977, Nature, 269, 112

Finger, M. H., Wilson, R. B., \& Chakrabarty, D. 1996a, A\&AS, 120C, 209

Finger, M. H., Wilson, R. B., \& Harmon, B. A. 1996b, ApJ, 459, 288

Fishman, G. J., et al. 1989, Proc. Gamma-Ray Observatory Science Workshop, ed. W. N. Johnson (Greenbelt, MD: NASA GSFC), 2

Hansen, B. M. S., \& Phinney, E. S. 1997, MNRAS, 291, 569

Hanuschik, R. W. 1996, A\&A, 308, 170

Harmon, B. A., et al. 1992, in Compton Observatory Science Workshop, ed. C. R. Schrader, N. Gehrels, \& B. Denis (NASA CP 3137) (Greenbelt, MD: NASA), 69

Hayakawa, S., Ito, K., Matsumoto,T., \& Uyama, K. 1977, A\&A, 58, 325

Johnston, S., Manchester, R. N., Lyne, A. G., Bailes, M., Kaspi, V. M., Guojun, Q., \& D’Amico, N. 1992, ApJ, 387, L37

Kalogera, V. 1996, ApJ, 471, 352

Kaspi, V. M., Bailes, M., Manchester, R. N., Stappers, B. W., \& Bells, J. F. 1996, Nature, 381, 584

Kaspi, V. M., Johnston, S., Bell, J. F., Manchester, R. N., Bailes, M., Bessel, M., Lyne, A. G., \& D'Amico, N. 1994, ApJ, 423, L43

Koyama, K., Kawada, M., Kunieda, H., Tawara, Y, Takeuchi, Y., \& Yamauchi, S. 1990a, Nature, 343, 148

\section{REFERENCES}

Koyama, K., Kunieda, H., Takeuchi, Y., \& Tawara, Y. 1990b, PASJ, 42, L59

Lai, D., Bildsten, L., \& Kaspi, V. M. 1995, ApJ, 452, 819

Makino, F., et al. 1988, IAU Circ. 4661

Press, W. H., Teukolsky, S. A., Vetterling, W. T., \& Flannery, B. P. 1992, Numerical Recipes in Fortran (2d ed.; Cambridge: Cambridge Univ. Press)

Quirrenbach, A., et al. 1997, ApJ, 479, 477

Ruffert, N. 1997, A\&A, 317, 793

Slettebak, A. 1988, PASP, 100, 770

Soffitta, P., Tomsick, J. A., Harmon, B. A., Costa, E., Ford, E. C., Tavani, M., Zhang, S. N., \& Kaaret, P. 1998, ApJ, 494, L203

Stollberg, M. T., Finger, M. H., Wilson, R. B., Scott, D. M., Crary, D. J., \& Paciesas, W. S. 1999, ApJ, 512, 313

Waters, L. B. F. M., de Martino, D., Habets, G. M. H. J., \& Taylor, A. R. 1989, A\&A, 223, 207

Wilson, C. A., Finger, M. H., Harmon, B. A., Scott, D. M., Wilson, R. B., Bildsten, L., Chakrabarty, D., \& Prince, T. A. 1997, ApJ, 479, 388

Wood, K. S., Bjorkman, K. S., \& Bjorkman, J. E. 1997, ApJ, 477, 926

Wood, K. S., et al. 1984, ApJS, 56, 507

van den Heuvel, E. P. J. 1994, in Interacting Binaries, ed. H. Nussbaumer \& A. Orr (Berlin: Springer), 263

Villa, G., Page, C. G., Turner, M. J. L., Cooke, B. A., Ricketts, M. J.,

Pounds, K. A., \& Adams, D. J. 1976, MNRAS, 176, 609

Zhang, S. N., et al. 1996, A\&AS, 120C, 227 